\title{
The acid-producing capacity and anti-microbial activity of Lactobacillus species isolated from patients treated for cancer in the head and neck region
}

\section{Matilda Rahne \\ Odontology}

\section{Amina Basic}

Goteborgs universitet Institutionen for odontologi

Annica Almståhl ( $\square$ annica.almstahl@odontologi.gu.se )

Goteborgs universitet Institutionen for odontologi https://orcid.org/0000-0003-1736-4452

\section{Research article}

Keywords: Lactobacillus species, tongue, plaque, acid-producing capacity, anti-microbial activity, head and neck cancer

Posted Date: May 9th, 2019

DOl: https://doi.org/10.21203/rs.2.9525/v1

License: (c) (1) This work is licensed under a Creative Commons Attribution 4.0 International License.

Read Full License 


\section{Abstract}

Background: Lactobacillus are acid-producing bacteria associated with caries development. Some Lactobacillus have also anti-microbial properties. In patients treated for cancer in the head and neck region, a marked increase in Lactobacillus has previously been reported. The consequences of this ecological shift towards a Lactobacillus friendly environment are, however, still unknown. Little is known about the prevalence of different Lactobacillus species and their characteristics in these patients. The aim of this study was therefore to identify Lactobacillus isolates from patients with head and neck cancer and to determine their acid-producing capacity and anti-microbial activity. Methods: Lactobacillus isolates were collected from 21 patients pre-treatment, six and 12 months post treatment. For identification to species level, the isolates were subjected to DNA-extraction, amplification of $16 \mathrm{~S}$ and restriction fragment length polymorphism analysis using the restriction enzymes Hpa II and Hae III. The acid-producing capacity was analyzed using a colorimetric fermentation test in microtiter plates. The anti-microbial activity of the isolates against Streptococcus mutans associated with caries, and the mucosal pathogens Staphylococcus aureus, Candida albicans and Enterococcus faecalis was determined by determining inhibitory zones on agar plates. Results: The most frequent species were $\mathrm{L}$. paracasei $(n=21)$, L. casei/rhamnosus $(n=17)$ and L. fermentum $(n=10)$. The highest proportion showing acid-producing capacity using sugars and sugar-alcohols was found among $\mathrm{L}$. paracasei and the lowest proportions were seen for L. fermentum. Seventy-one strains showed an anti-microbial activity against $\mathrm{S}$. mutans, one strain against $\mathrm{S}$. aureus and none against $\mathrm{C}$. albicans or E. faecalis. Conclusion: The most frequent species Lactobacillus species isolated from patients with head and neck cancer were $L$. paracasei, L. casei/rhamnosus and L. fermentum of which L. paracasei showed the best acid-producing capacity. A weak anti-microbial activity against $\mathrm{S}$. mutans was frequently detected. Further studies are needed to increase the knowledge of the role and characteristics of oral Lactobacillus.

\section{Background}

Lactobacillus are Gram-positive rod-shaped bacteria that are generally facultatively anaerobic, with a range from microaerophilic to strictly anaerobic. Lactobacillus species belong to the normal flora in the gastro-intestinal tract and in the female genitourinary tract [1,2]. Lactobacillus can either be homofermentative or heterofermentative. Homofermentative species use glucose, which is almost completely degraded to lactic acid. Heterofermentative Lactobacillus degrade glucose to carbon dioxide, acetic acid, ethanol and lactic acid.

In the oral cavity of individuals with good oral health, Lactobacillus are rarely detected, while their proportion of the total count is approximately $10 \%$ in irradiated subjects [3]. In subjects with good oral health, L. plantarum, L. rhamnosus, L. salivarius, L. acidophilus and $L$. gasseri are frequently found species [4-6]. Most of these species have also been found in subjects with caries; $L$. fermentum, $L$. plantarum, L. rhamnosus, L. casei, L. delbrueckii, L. salivarius, L. vaginalis and L. brevis $[5,7-8]$. In 
subjects with hyposalivation due to either primary Sjögren's syndrome or to radiation therapy (3-5 years post treatment) the most prevalent species were L. fermentum, L. casei and L. rhamnosus [9].

The ability to produce acids is a common trait among the Lactobacillus spp. [10]. There are, however, variations between different species [10] and also between strains of the same species. In a previous in vitro study our group showed that $L$. rhamnosus could lower the $\mathrm{pH}$ to between 4.2 and 4.7 using glucose, fructose and sucrose and to $\mathrm{pH}$ 5.1-5.6 using the sugar alcohols mannitol, sorbitol and xylitol. $L$. fermentum could not lower the $\mathrm{pH}$ as much, but to between $\mathrm{pH} 4.8-5.8$ using sugars and between $\mathrm{pH}$ 6.46.5 using sugar alcohols [11]. In a recent study, the acid-producing capacity of 211 Lactobacillus strains isolated from patients with cancer in the head and neck region pre-treatment, during treatment and six and 12 months post treatment was analyzed. The number of isolates that showed a strong acidproducing capacity using sugars was highest for the isolates collected during treatment and six months post treatment [12]. At 12 months post treatment, the number of isolates with strong acid-producing capacity had decreased but was still higher compared with pre-treatment. For sorbitol, none of the isolates showed a strong acid-producing capacity at pre-treatment, while $24 \%$ had this ability at 12 months post treatment. The acid-producing capacity using xylitol was also more frequent among isolates collected during treatment and at 12 months post treatment the number of isolates that showed weak acid-producing capacity using xylitol was slightly higher compared with pre-treatment [12]. Sugaralcohols are commonly used in products to relieve dry mouth like for example chewing gum and tablets. It is likely that frequent use of such products can promote the growth of Lactobacillus that are able to ferment sugar-alcohols.

The role of the increased number of Lactobacillus species found in the oral cavity of hyposalivated subjects is not fully understood. On the one hand, Lactobacillus contribute to an acidic environment, which is undesireable for microorganisms associated with good oral health such as for example streptococci and Neisseria. On the other hand, Lactobacillus spp. have been demonstrated to have antimicrobial effects. The antimicrobial activity of $L$. casei rhamnosus against several pathogens such as for example Escherichia coli, Enterobacter cloacae and Salmonella typhimurium has been reported [13]. Furthermore, a study on the bacteriocin production of Lactobacillus species isolated from plaque and saliva of patients with caries showed that all species except $L$. jensenii produced bacteriocins against at least one bacteria among E. coli, Salmonella spp, Klebsiella, Shigella dysenteriae, S. sonnei and Campylobacter [7]. Other studies have also shown that Lactobacillus (strains of L. casei, L. fermentum, L. plantarum, L. paracasei, L. salivarius, L. rhamnosus) can have anti-microbial effects on Streptococcus mutans [6, 14-17]. The majority of Lactobacillus strains isolated from saliva and subgingivally from healthy subjects were able to suppress the growth of the periodontal pathogens Aggregatibacter actinomycetemcomitans, Porphyromonas gingivalis, and Prevotella intermedia [16]. In addition, ingestion 
of cheese containing several probiotic bacteria of which two were Lactobacillus strains (L. rhamnosus GG and LC705) reduced high levels of Candida in elderly subjects [18].

Knowledge about the prevalence of different Lactobacillus species in the oral cavity of patients subjected to radiation therapy to the head and neck region is limited and little is known about their acid-producing capacity and anti-microbial activity. Such knowledge is valuable to understand the role of elevated numbers of Lactobacillus species in the oral cavity.

The aim of this study was to identify Lactobacillus isolates from patients with head and neck cancer and to determine the acid-producing capacity and anti-microbial activity of the isolates.

\section{Methods}

Microbial samples had been collected from 33 patients ( 23 men, 10 women, mean age $59 \pm 8$ years and the mean number of teeth $25 \pm 5$ teeth) included in the project entitled "Longitudinal changes in saliva, microflora, diet and oral status in relation to the quality of life in people receiving radiotherapy to the head and neck region". The project has been approved by the Ethical Committee at the University of Gothenburg (Dnr 682-07). The microbial samples were collected at pre-treatment, and at six and 12 months post treatment at the Department of Oral and Maxillofacial Surgery, Gothenburg. The patients' clinical status, minor and major salivary secretion rates and microflora has been reported previously [31, 32].

\section{Microbial sampling}

The sites sampled were the dorsum of the tongue, buccal mucosa (bilaterally) and supragingival plaque. The methods for microbial sampling have been described previously [12]. The bottles with VMGA III were shaken on a Whirlymixer for $10 \mathrm{~s}$ and thereafter $0.1 \mathrm{ml}$ was placed and stroked in a standardized fashion to Rogosa agar plates (Neogen, MI, US) [33], which were incubated in $36^{\circ} \mathrm{C}$ for $5-7$ days.

The microorganisms were semi-quantified according to Dahlén et al., [34] based on their typical colony morphology. The growth was registered as no growth, very sparse ( $<10$ colonies), sparse $(>10-100$ colonies), moderate (growth also in the second stroke) and heavy (growth also in the third stroke). From their growth on Rogosa agar plates, the most dominating colony types (1-2 types) were isolated and Gram-stained to verify that they were Gram-Positive rods. Thereafter the isolates were recultivated until purity and were then stored at $-80^{\circ} \mathrm{C}$. All Lactobacillus isolates (from 21 different patients), which had 
showed moderate or heavy growth at pre-treatment and at six and 12 months post treatment $(n=66)$ were selected for this study.

\section{Bacterial identification}

Lactobacillus isolates were cultured on Rogosa agar plates, which had been incubated in $90 \% \mathrm{~N}_{2}$ and $10 \% \mathrm{CO}_{2}$ at $36^{\circ} \mathrm{C}$ for $3-5$ days. Four of the isolates grew poorly and were therefore excluded. For the remaining 62 isolates, bacterial colonies corresponding to approximately $1 \mathrm{ml}$ were harvested. The isolates were treated with Fermentas GeneJet Genomic DNA Purificarion Kit (ThermoFisher Scientific, Sweden) using their protocol for Gram positive bacteria. For 15 of the 62 samples a too low level of genetic material was obtained and a more extensive DNA purification protocol was used as described by Teanpaisan and Dahlén [30].

\section{Polymerase Chain Reaction (PCR)}

The PCR protocol used to amplify $16 \mathrm{~S}$ was based on a previous study [31]. Briefly, four microliters of genetic material (sample) containing approximately $100 \mathrm{ng}$ template DNA was mixed with the primers (8UA and 1492R) and HotMasterMix (5 PRIME, Germany). The samples were treated during 35 cycles of denaturation, annealing and extension followed by a final extension. The samples were then stored at $-20^{\circ} \mathrm{C}$.

Restriction fragment length polymorphism (RFLP)

The restriction enzymes used were Hpa II and Hae III (Thermo Scientific). The genetic material from the PCR was treated according to instructions from the manufacturer. To the wells of one $5 \%$ MiniPROTEAN ${ }^{\circledR}$ TBE Gel (Bio-Rad, USA) $8 \mu \mathrm{l}$ of samples treated with Hae III / Hpa II and mixed with $2 \mu \mathrm{l}$ of sample buffer was added and to wells of another gel. Nine wells were used for samples and one for a Molecular ruler (AmpliSize ${ }^{\circledR}$ Molecular Ruler, 50-2000 base pairs, BioRad). The electrophoresis was run at $100 \mathrm{~V}$ for $50 \mathrm{~min}$. The gel was then stained with a Silver staining kit (Plus One ${ }^{\mathrm{TM}}$, DNA Silver Staining Kit, GE Healthcare, Sweden) and dried with Drying Solution (Invitrogen, USA).

\section{Lactobacillus species identification}

The patterns of the strains on the dried gels were compared with the patterns on gels of 13 type strains (Table 1) included in a previous study [9]. 
Acid producing capacity

Lactobacillus isolates were cultivated on Rogosa agar plates. After $48 \mathrm{~h}$ of incubation, one colony was transferred to broth (Brain Heart Infusion $\left(\mathrm{BHI}-\right.$ Bacto $^{\mathrm{TM}}$ ). The tubes were incubated overnight in $90 \% \mathrm{CO}_{2}$ and $10 \% \mathrm{~N}_{2}$, at $36{ }^{\circ} \mathrm{C}$ and harvested at the mid-exponential phase. On the day of the experiment, the cultured bacterial cells were centrifuged for $5 \mathrm{~min}$ at $1300 \mathrm{~g}$ and the pellet washed twice with Phosphate buffered saline (PBS) and diluted to an optical density $(\lambda=650 \mathrm{~nm})$ of 1.0. A slightly modified version of the fermentation assay, described by Hedberg et al., [35], was used to test the acid production from sugars and sugar alcohols. The methodology has been described previously [12]. The acid-producing ability was determined by then use of a visual 3-graded scale as yellow (strong acid-producing capacity, $\mathrm{pH}<5$ ), red/brown (weak acid-producing capacity, $\mathrm{pH} \geq 5-\leq 6$ ), or purple (low/absent acid-producing capacity, $\mathrm{pH}>6$ ).

\section{Antimicrobial activity}

Four oral pathogens were selected as target microorganisms: S. mutans (OMGS 2482), Candida albicans (OMGS 3750), Staphylococcus aureus (OMGS 3871), and Enterococcus faecalis (OMGS 3632). S. mutans is associated with caries and $C$. albicans, $S$. aureus and $E$. faecalis are associated with oral mucosal infections. All of these species are found in increased levels in irradiated patients [3, 33]. The method used for testing the antimicrobial activity was based on previous experiments at the Department of Oral Microbiology and Immunology [20]. Lactobacillus isolates were cultivated on Rogosa agar for 48-72 h. Target microorganisms were cultivated on blood agar during $48 \mathrm{~h}$ at $36^{\circ} \mathrm{C}$ and then in Brain Heart infusion broth (BHI broth) over night. From the BHI broth with an optical density (OD) ${ }_{600}$ of $0.8-1.0,0.5 \mathrm{ml}$ was mixed with melted $\mathrm{BHI}$-agar and let to solidify for $20 \mathrm{~min}$. After which the Lactobacillus isolates were spread over an area of about $1.5 \mathrm{~cm}^{2}$. The plates were incubated for $48 \mathrm{~h}$ in an atmosphere appropriate for each target microorganism. Inhibition was determined visually as no inhibition (no clear zone), weak inhibition (clear zone of $\leq 1 \mathrm{~mm}$ ) or strong inhibition (clear zone $>1 \mathrm{~mm}$ ).

\section{Statistical methods}

In four cases two isolates from the same time-point and site had been collected. In three of these cases, they showed an identical acid-producing capacity pattern and it was therefore determined that the isolates belonged to the same species. Only one of the isolates was therefore used in the analysis and the number of isolates was reduced from 62 to 59 .

\section{Results}


As can be seen in Table 2, Lactobacillus isolates were collected from 21 patients, 15 men and six women with a mean age of $59 \pm 8$ years. The most common diagnoses were tonsil cancer (10 patients) followed by tongue base cancer ( 5 patients). The most common treatment modality was external radiation therapy (10 patients) combined with chemotherapy (10 patients) followed by both external radiation therapy and brachytherapy (iridium-implant) and also chemotherapy (7 patients). Seventeen of the patients were treated with intensity-modulated radiotherapy (IMRT) and four patients with conventional radiotherapy. The mean stimulated salivary secretion rate pre-treatment was $1.8 \pm 0.9 \mathrm{ml} / \mathrm{min}$ (median $1.9 \mathrm{ml} / \mathrm{min}$ ). The corresponding values at six, and 12 months post treatment was $0.5 \pm 0.5 \mathrm{ml} / \mathrm{min}(0.4 \mathrm{ml} / \mathrm{min})$ and $0.7 \pm 0.5 \mathrm{ml} / \mathrm{min}(0.6 \mathrm{ml} / \mathrm{min})$. The number of patients with hyposalivation $(\leq 0.7 \mathrm{ml} / \mathrm{min})$ was four (19\%) at pre-treatment, $15(71 \%)$ at six months post treatment and $13(62 \%)$ at 12 months post treatment.

Fifty-eight of the 59 Lactobacillus isolates were identified to species level. Only one isolate was neither of the 13 type strains included. Eight different Lactobacillus spp. were identified. The most common species were L. paracasei $(n=21)$ followed by L. casei/rhamnosus $(n=17)$, L. fermentum $(n=10)$ and L. plantarum $(n=4)$. Eight of the Lactobacillus isolates had been collected pre-treatment, 24 at six months posttreatment and 27 at 12 months post-treatment.

In Table 3 the isolates collected from the tongue and plaque at pre-treatment, at six, and 12 months post treatment for each patient is shown. Only five isolates came from the buccal mucosa and are therefore not shown. L. paracasei were slightly more frequently found at 12 months post treatment (tongue; $n=5$, plaque $n=7$ ) compared with pretreatment (tongue; $n=2$ ) and six months post treatment (tongue; $n=4$, plaque $n=4$ ). The prevalence of $L$. fermentum was highest at six months post treatment (Table 3 ).

\section{Acid-producing capacity irrespective of time-point for collection}

The proportion of isolates that showed strong acid-producing capacity by the use of sucrose, glucose, fructose and lactose was highest among the $L$. paracasei isolates (43-76\%), while the lowest proportion was seen for the L. fermentum isolates (20-30\%) (Fig 1a). Strong acid-producing capacity using sorbitol was only found for L. paracasei (19\%) (Fig 1b). The proportion of isolates showing weak acid-producing capacity using sorbitol was higher for the L. paracasei and L. casei/rhamnosus isolates than for the $L$. fermentum isolates (Fig 1b). A similar pattern was seen for weak acid-producing capacity using xylitol (Fig 1 b). Among the 11 remaining isolates, the proportion showing strong acid-producing capacity was highest for fructose (56\%). The proportion that showed weak acid-producing capacity using sorbitol and xylitol was $36 \%$ for both. It should be noted that the acid-producing capacity varied between different isolates within the same species and in most cases isolates with strong, weak and no acid-producing capacity were found. 
Acid-producing capacity using sugars for strains isolated at different time-points

The proportion of $L$. paracasei isolates showing strong acid-producing capacity using sugars was lower among isolated collected at 12 months post treatment compared with those collected pre-treatment and six months post treatment (Fig 2a). For L. casei/rhamnosus there was a markedly higher proportion showing strong acid-producing using glucose and fructose among isolates collected at six months post treatment compared with those collected pre-treatment. L. fermentum showed a different pattern with the highest proportion showing strong acid-producing capacity seen among isolates collected at 12 months post RT (Fig 2a).

Acid-producing capacity using sorbitol for strains isolated at different time-points

For L. paracasei the proportion of isolates showing weak acid-producing capacity was $100 \%$ at pretreatment and $75 \%$ at 12 months post treatment (Fig 2b). Among L. casei/rhamnosus isolates, all showed weak acid-producing capacity at six months post treatment and the corresponding figure at 12 months post RT was $58 \%$. None of the $L$. fermentum isolates collected at six months post treatment were able to produce acids using sorbitol, while $50 \%$ had that ability at 12 months post treatment (Fig 2 b).

Acid-producing capacity using xylitol at different time-points

The proportion of $L$. paracasei showing weak acid-producing capacity using xylitol was highest among isolates collected at six months post treatment (71\%) (Fig 2b). Also for L. casei/rhamnosus, the highest proportion that showed weak acid-producing capacity was seen among isolates collected at six months post treatment (88\%) (Fig 2b). The proportion of L. fermentum isolates that showed weak acid-producing capacity using xylitol was slightly higher among isolates collected at 12 months post treatment $(50 \%)$ compared with those isolated at pre-treatment (33\%), while no L. fermentum isolates collected at six months post treatment were able to produce acids using xylitol (Fig 2b).

\section{Anti-microbial activity}

None of the 59 isolates showed anti-microbial activity against $C$. albicans or $E$. faecalis and only one strain against $S$. aureus (an L. acidophilus isolate collected from the tongue 12 months post treatment showed strong anti-microbial activity). Forty-two isolates (71\%) showed an anti-microbial activity against S. mutans, 40 showed weak anti-microbial activity and two isolates strong. In Table 4, the anti-microbial activity for the 58 isolates is shown. The highest proportion of isolates with anti-microbial activity against S. mutans was seen for L. paracasei $(81 \%)$ followed by L. fermentum $(70 \%)$ and L. casei/rhamnosus 
(56\%). There was no clear correlation between growth of $S$. mutans in the supragingival plaque and presence of Lactobacillus with anti-microbial activity. Both patients with no growth and patients with heavy growth of $S$. mutans harboured Lactobacillus with anti-microbial activity against $S$. mutans. The highest proportion with anti-microbial activity was seen among isolates collected at pre-treatment $86 \%$ ( 6 of 7 isolates) followed by six months post treatment $71 \%$ (17 of 24 isolates) and lastly at 12 months post treatment $67 \%$ ( 18 of 27 isolates).

\section{Discussion}

In the present study, Lactobacillus spp. collected from the buccal mucosa, tongue and plaque of 21 patients treated for cancer in the head and neck region were identified to species level and their acidproducing capacity, and anti-microbial activity was determined. The most prevalent species were $L$. paracasei, $L$ casei/rhamnosus and $L$. fermentum of which $L$. paracasei was the most prominent species at 12 months post treatment. The highest proportion of isolates that showed strong acid-producing capacity using sugars was found for $L$. paracasei of which four showed strong acid-producing capacity using sorbitol. The proportion that showed weak acid-production capacity using xylitol was highest among L. casei/rhamnosus both at six, and 12 months post treatment. Anti-microbial activity was seen for one isolate against $S$. aureus and for 42 isolates $(71 \%)$ against $S$. mutans. All but one patient had Lactobacillus with antimicrobial activity against $S$. mutans at least at one time-point. Antimicrobial activity was most prevalent among $L$. paracasei (81\%).

In our previous study, using the same method for identification, the most frequently isolated species from plaque in irradiated patients 3-5 years post treatment were L. fermentum $29 \%$, L. casei $22 \%$, L. rhamnosus $19 \%$ and $L$. paracasei $7 \%$ [9]. In the present study, a slightly lower prevalence of $L$. casei/rhamnosus was found, $33 \%$. The prevalence of $L$. paracasei was much higher, $53 \%$, compared with the previous study. There was also a difference in the prevalence of $L$. fermentum since no $L$. fermentum were isolated from the plaque at 12 months post RT in the present study. A possible explanation to the discrepancies might be that in the former study several isolates were collected from either anterior or posterior tooth surfaces [9], while in the present study only 1-2 isolates was collected from samples where plaque from four sites had been pooled.

To the best of our knowledge, there are no other previous reports on the prevalence of different Lactobacillus species in the oral cavity and their acid-producing capacity at different time-points in relation to treatment for cancer in the head and neck region. 
The results of the present study suggest that there might be differences in acid-producing capacity among Lactobacillus isolated at different time-points. For some species a lower proportion showing strong acid-producing capacity was seen post treatment, while others showed a higher proportion. With the method used for identification it is not possible to determine whether the same strain was isolated at different time-points or if it were other strains. It is possible that the persistent acid environment due to the reduced salivary secretion rate enhanced the growth of certain Lactobacillus strains and species more than others.

\section{Acid-producing capacity and species}

In accordance with our previous study [9], the proportion of L. fermentum isolates that showed strong acid-producing capacity using sugars and sugar-alcohols was lower compared with $L$. paracasei and $L$. casei/rhamnosus. A high proportion of the $L$. paracasei isolates was able to decrease the $\mathrm{pH}$ to $<5.0$ using sucrose, glucose and fructose, which is also in accordance with the results of our previous study [9]. It has been shown that it takes a longer time for the $\mathrm{pH}$ to reach 5.5 using glucose for $L$. fermentum, $4.18 \mathrm{~h}$, compared with L. casei/paracasei, $2.87 \mathrm{~h}$, and L. rhamnosus $2.27 \mathrm{~h}[19$ ]. Also, the final pH is higher for $L$. fermentum, $\mathrm{pH} 4.58$, compared with $L$. casei/paracasei, $\mathrm{pH} 4.02$ and $L$. rhamnosus $\mathrm{pH} 3.89$ [19]. In our previous study, the proportion of strains that were able to produce acids using sorbitol was highest among $L$. paracasei (49\%), while L. fermentum rarely had that ability [9]. These results are also in line with those in the present study where $19 \%$ of the $L$. paracase $i$ isolates showed a strong acidproducing capacity using sorbitol and $66 \%$ weak; while only $20 \%$ of the $L$. fermentum isolates had this ability (Fig 2b).

\section{Antimicrobial activity}

In the present study, anti-microbial activity was determined on agar plates where growth inhibition of the test microorganism was registered. This method has been used for the determination of the antimicrobial activity for enterococcus strains at out laboratory [20]. Lactobacillus spp. can have several activities or abilities, which can contribute to inhibition of other species such as bacteriocins, bi-products of fermentation like lactic acid and hydrogen peroxide, or their ability to reduce the $\mathrm{pH}$ [1]. Due to the method used for the determination of possible inhibition in the present study, no information about which activities or abilities that were responsible for the effect can be obtained. Further studies are therefore needed to elucidate the mechanisms involved in this inhibitory effect.

All but one patient had Lactobacillus with anti-microbial activity against $S$. mutans at least at one timepoint. A high proportion of the Lactobacillus isolates, $71 \%$, had an anti-microbial activity against $S$. mutans of which $3 \%$ showed strong activity and $68 \%$ weak. High proportions of Lactobacillus with anti- 
microbial activity against $S$. mutans have previously been reported also in other studies $[6,16,21]$. Twenty-five percent of the Lactobacillus isolates collected from caries-free and caries-active children and young adults were able to completely inhibit $S$. mutans, while $75 \%$ showed no or low inhibition [21]. Among Lactobacillus isolates collected from both healthy persons and periodontitis patients, the proportion with ability to inhibit S. mutans was $69 \%[16]$ and in another study including 67 Lactobacillus isolates, $55 \%$ could inhibit $S$. mutans [6]. In the present study, most isolates showed weak anti-microbial activity, thus a possible inhibitory effect on $S$. mutans in vivo is questionable.

Lactobacillus spp. from patients without caries experience have been shown to have a higher inhibitory effect on S. mutans than Lactobacillus from patients with arrested or active caries [22]. In the present study, we found no clear correlation between presence of Lactobacillus spp. with anti-microbial activity against $S$. mutans, and growth of $S$. mutans. No correlation with caries status was found. This lack of correlation may be due to the relatively low number of patients included in the present study. Other plausible explanations may be that also other bacteria are involved in caries development like for example Scardovia wiggsiae and Bifidobacterium dentium [23, 24]. In addition, other factors are involved in the caries process such as the amount and quality of saliva, intake frequency of easily fermentable carbohydrates, oral hygiene level, and fluoride exposure [25].

Species able to inhibit $S$. mutans have previously been reported among L. plantarum, L. paracasei, $L$. salivarius, $L$. rhamnosus and $L$. fermentum, but the proportion of the isolates having this ability were not shown [16]. Similar results have also been shown by Simark-Mattsson et al., [21] where the highest proportions of the strain able to inhibit $S$. mutans were found for $L$. paracasei (47\% of the strains), $L$. plantarum (30\%), and L. rhamnosus (23\%). In the present study, the highest proportion of isolates with anti-microbial activity against $S$. mutans was also found among L. paracasei, $81 \%$. However, in the present study a high proportion of $L$. fermentum also showed anti-microbial activity, $70 \%$, while the one $L$. fermentum included in the study by Simark-Mattsson et al., [21] could not inhibit $S$. mutans. A plausible explanation for the divergent results is that different populations were included, Simark-Mattsson et al., [21] isolated Lactobacillus from children and young adults, while in the present study Lactobacillus were isolated from patients with a mean age of 59 years who had undergone treatment for cancer in the head and neck region, and therefore presented a specific oral environment.

Only one of the 59 isolates, an L. acidophilus strain collected from the tongue at 12 months post treatment, showed an anti-microbial activity against $S$. aureus and the patient showed no growth of $S$. aureus at any of the sampling sites or time-points. Among the patients, the detection frequencies of $S$. aureus were $57 \%$ at six months post treatment and $43 \%$ at 12 months post treatment. It is not unusual 
that $L$. acidophilus have this anti-bacterial activity against $S$. aureus, all of eight previously tested strains were able to inhibit $S$. aureus and seven of them had a bactericidal effect [26].

In the present study, none of the isolates showed an anti-microbial activity against $C$. albicans, which is in congruence with Köll et al., [16], who reported that none of the 67 Lactobacillus isolates from healthy persons had any anti-microbial activity against $C$. albicans. In another study where the anti-microbial activity of Lactobacillus isolates from saliva of healthy volunteers was tested, it was found that two out of four $L$. paracasei sp paracasei were able to inhibit the growth of two out of three $C$. albicans strains. Furthermore one of two L. rhamnosus strains inhibited two out of the three $C$. albicans strains [27]. It is difficult to explain the divergent results, but it is possible that the ability to inhibit $C$. albicans is strainspecific. Both Lactobacillus and C. albicans are frequently found in patients after cancer treatment [28], which implies that the Lactobacillus that these patients harbour do not inhibit $C$. albicans. Another explanation might be that the acidic environment that is present in many subjects with reduced salivary secretion rate after cancer treatment is a stronger promoting factor for the growth of $C$. albicans. $C$. albicans were detected in 13 of the 21 patients (62\%) at six months post treatment and in 15 of $21(71 \%)$ at 12 months post treatment.

None of the isolates in the present study showed an anti-microbial activity against $E$. faecalis. Two probiotic strains, an L. plantarum and an L. rhamnosus have previously been shown to inhibit an $E$. faecalis strain [29]. It is possible that the ability to inhibit or have an anti-microbial activity against $E$. faecalis is not a common trait among wild Lactobacillus strains, especially not among oral Lactobacillus since enterococci are not part of the resident oral microflora. Enterococci were detected in two of the 21 patients (9\%) at six months post treatment and in only one patient at 12 months post treatment.

\section{Methodological considerations}

With the method used $L$. casei and $L$. rhamnosus could not be distinguished since their patterns on the gel was identical. To differentiate between these two species, they could have been subjected to sodium dodecyl sulphate-polyacrylamide gel electrophoresis (SDS-PAGE) for whole cell protein analysis $[9,30]$. However, little is known about differences between these two species and it is unlikely that such information would have added much to the results.

\section{Conclusions}

In patients who have been treated for cancer in the head and neck region the prevalence of oral Lactobacillus spp. varies, depending on the site sampled and the time-point of collection. The most frequent species were L. paracasei, L. casei/rhamnosus and L. fermentum. The highest proportion of 
isolates that showed a strong acid-producing capacity using both sugars and sugar-alcohols was seen for $L$. paracasei. A weak anti-microbial activity against $S$. mutans was seen for $71 \%$ of the isolates. Further studies are needed to elucidate the role of the highly elevated numbers of Lactobacillus spp. In patients treated for cancer in the head and neck region.

\section{Declarations}

Acknowledgements: Not applicable

Ethical approval and consent to participate: In the present study, Lactobacilli collected from patients with cancer in the head and neck region was characterised. The main project including for example clinical examinations and microbial sampling has been approved by the Ethical Committee at the University of Gothenburg (Dnr 682-07). All patients signed an informed consent.

Consent for publication: Not applicable

Availability of data and material: The datasets used and/or analysed during the current study are available from the corresponding author on reasonable request.

Competing interests: The authors declare that they have no competing interests.

Funding: This study was supported by grants from The Health \& Medical Care Committee of the Regional Executive Board, Region Västra Götaland, and TUA research Gothenburg. The funding bodies had no role in the design of the study, analysis, interpretation of data or in writing the manuscript.

Authors' contribution: Authors MR and AA designed the study. Author MR performed the identification of Lactobacillus isolates and author $A B$ performed analysis of acid-producing capacity and anti-microbial activity of the Lactobacillus isolates. All authors participated in the analysis of the data and writing of the manuscript. All authors read and approved the final version of the manuscript.

Acknowledgements: Not applicable 


\section{References}

1. Slover CM, Danziger L. Lactobacillus: a review. Clin Microbiol Newsletter. 2008;30:23-27.

2. Caufield PW, Schön CN, Saraithong P et al. Oral lactobacilli and dental caries: a model for niche adaptation in humans. J Dent Res. 2015;94(9 suppl):100S-118S.

3. Almståhl A, Wikström M, Fagerberg-Mohlin B. Microflora in oral ecosystems in subjects with radiation-induced hyposalivation. Oral Dis. 2008;14:541-549.

4. Colloca ME, Ahumada MC, Lopez ME et al. Surface properties of lactobacilli isolated from healthy subjects. Oral Dis. 2000;6:227-233.

5. Ahumada MC, Bru E, Colloca ME et al. Evaluation and comparison of lactobacilli characteristics in the mouths of patients with or without cavities. J Oral Sci. 2003;45:1-9.

6. Köll-Klais P, Mändar R, Leibur E et al. Oral lactobacilli in chronic periodontitis and periodontal health: species composition and antimicrobial activity: Oral Microbiol Immunol. 2005;20:354-361.

7. Smith $\mathrm{SI}$, Aweh AJ, Coker AO et al. Lactobacilli in human dental caries and saliva. Microbios. 2001;105:77-85. 
8. Caufield PW, Li Y, Dasanayake A et al. Diversity of Lactobacilli in the Oral Cavities of Young Women with Dental Caries. Caries Res. 2007;41:2-8.

9. Almståhl A, Carlén A, Eliasson L et al. Lactobacillus species in supragingival plaque in subjects with hyposalivation. Arch Oral Biol. 2010;55:255-259.

10. Piwat S, Teanpaisan R, Thitasomakul S et al. Lactobacillus species and genotypes associated with dental caries in Thai preschool children. Molec Oral Microbiol. 2010;25:157-164.

11. Almståhl A, Lingström P, Eliasson L, Carlén A. Fermentation of sugars and sugaralcohols by plaque Lactobacillus species. Clin Oral Invest. 2013;17:1465-1470.

12. Almståhl A, Rudbäck H, Basic $\mathrm{C}$ et al. Acid producing capacity from sugars and sugar alcohols among Lactobacillus isolates collected in connection with radiation therapy. Arch Oral Biol. 2017;84:82-88.

13. Forestier C, De Champs C, Vatoux C et al. Probiotic activities of Lactobacillus casei rhamnosus: in vitro adherence to intestinal cells and antimicrobial properties. Res Microbiol. 2001;152:167-173.

14. Näse L, Hatakka K, Savilahti E et al. Effect of long-term consumption of a probiotic bacterium, Lactobacillus rhamnosus GG, in milk on dental caries and caries risk in children. Caries Res. 2001;35:412-420. 
15. Ahola AJ, Yli-Knuutila H, Suomalainen et al. Short-term consumption of probioticcontaining cheese and its effect on dental caries risk factors. Arch Oral Biol. 2002;41:799-804.

16. Köll P, Mändar R, Marcotte $H$ et al. Characterization of oral lactobacilli as potential probiotics for oral health. Oral Microbiol Immunol. 2008;23:139-147.

17. Keller MK, Hasslöf P, Stecksén-Blicks $C$ et al. Co-aggregation and growth inhibition of probiotic lactobacilli and clinical isolates of mutans streptococci: An in vitro study. Acta Odontol Scand. 2011;69:263-268.

18. Hatakka K, Ahola AJ, Yli-Knuuttila $\mathrm{H}$ et al. Probiotics reduce the prevalence of oral Candida in the elderly - a randomized controlled trial. J Dent Res. 2007;86:125130.

19. Piwat $\mathrm{S}$, Teanpaisan R, Dahlén $\mathrm{G}$ et al. Acid production and growth by oral Lactobacillus species in vitro. J Invest Clin Dent. 2012;3:56-61.

20. Dahlén G, Blomqvist S, Almståhl A et al. Virulence factors and antibiotic susceptibility in enterococci isolated from oral mucosal and deep infections. J Oral Microbiol. 2012; doi: 10.3402/jom v410.10855. 
21. Simark-Mattson C, Jonsson R, Emilson CG, Roos K. Final pH affects the Interference capacity of naturally occuring oral Lactobacillus strains against mutans streptococci. Arch Oral Biol. 2009;54:602-607.

22. Simark-Mattson C, Emilson CG, Håkansson EG et al. Lactobacillus-mediated interference of mutans streptococci in caries-free vs. Caries-active subjects. Eur J Oral Sci. 2007;115:308-314.

23. Henne K, Rheinberg A, Melzer-Krick B et al. Aciduric microbial taxa including Scarodiva wiggsiae and Bifidobacterium spp. in caries and caries-free subjects. Anaerobe. 2015;35:60-65.

24. Zhou J, Jiang N, Wang S et al. Exploration of human salivary microbiomesinsights into the novel characteristics of microbial community structure in caries and caries-free subjects. PlosOne. 2016; doi:10.1371/journal.pone.0147039.

25. Fejerskov O, Nyvad B, Kidd EAM. Dental caries: the disease and its clinical management (Vol 3) Chichester: Wiley-Blackwell.

26. Pyar H, Liong M-T, Peh KK. Characteristics and antibacterial activity of metabolites from Lactobacillus acidophilus strains produced from novel culture media. Int $\mathrm{J}$ Pharmacol. 2013;9:92-97.

27. Sookkhee S, Chulasiri M, Prachyabrued W. Lactic acid bacteria from healthy oral 
cavity of Thai volunteers: Inhibition of oral pathogens. J Appl Microbiol. 2001;90:172-179.

28. Almståhl A, Wikström M, Fagerberg-Mohlin B. Microflora in oral ecosystems and salivary secretion rates - A 3-year follow-up after radiation therapy in the head and neck region. Arch Oral Biol. 2015;60:1187-1195.

29. Bohora AA, Kokate SR. Good bugs vs bad bugs: evaluation of inhibitory effect of selected probiotics against Enterococcus faecalis. J Contempl Pract. 2017;18:312316.

30. Almståhl A, Finizia C, Carlén A et al. Mucosal microflora in head and neck cancer patients. Int J Dent Hyg. 2018a;16:459-466.

31. Almståhl A, Finizia C, Carlén A, Fagerberg-Mohlin B, Alstad. Explorative study on mucosal and major salivary secretion rates, caries and plaque microflora in head and neck cancer patients. Int J Dent Hyg 2018b;16:450-458.

32. Dahlén G, Linde A, Möller Å et al. A retrospective study of microbiological samples from oral mucosal lesions. Oral Surg Oral Med Oral Pathol. 1982;53:250-255.

33. Teanpaisan R, Dahlén G. Use of polymerase chain reaction techniques and sodium dodecyl sulfate-polyacrylamide gel electrophoresis for differentiation of oral Lactobacillus species. Oral Microbiol Immunol. 2006;21:79-83. 
34. Teanpaisan R, Hintao J, Dahlén G. Oral Lactobacillus species in type 2 diabetic Patients living in southern Thailand. Anaerobe. 2009;15:160-163.

35. Hedberg M, Hasslöf P, Sjöström I et al. Sugar fermentation in probiotic bacteria -an in vitro study. Oral Microbiol Immunol. 2008;23:482-485.

36. Eliasson L, Carlén A, Almståhl A et al. Dental plaque pH and microorganisms during hyposalivation. J Dent Res. 2006;85:334-338.

\section{Tables}

Due to technical limitations, the tables have been placed in the Supplementary Files section.

\section{Figures}




\section{$1 a$}

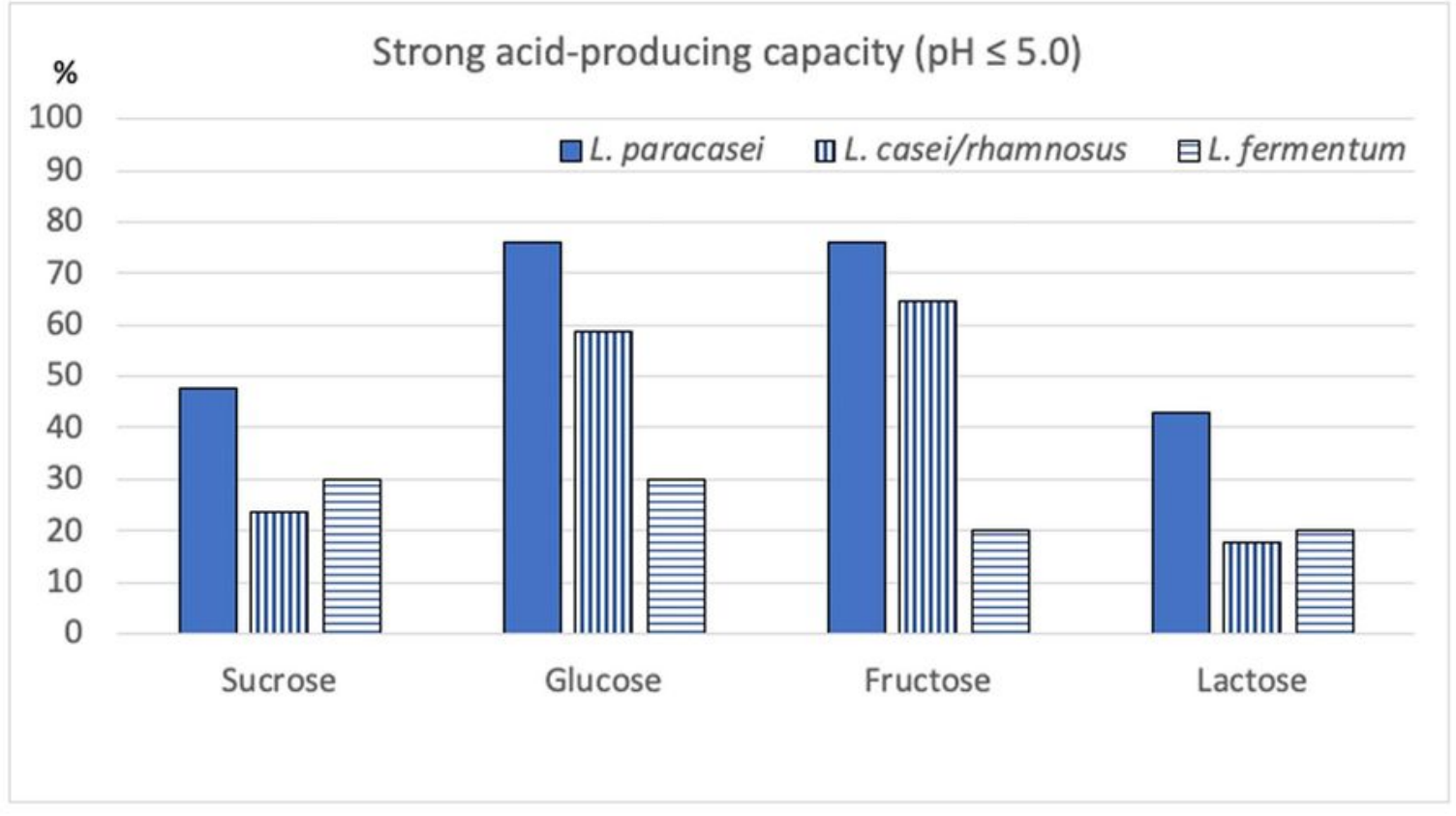

\section{$1 b$}

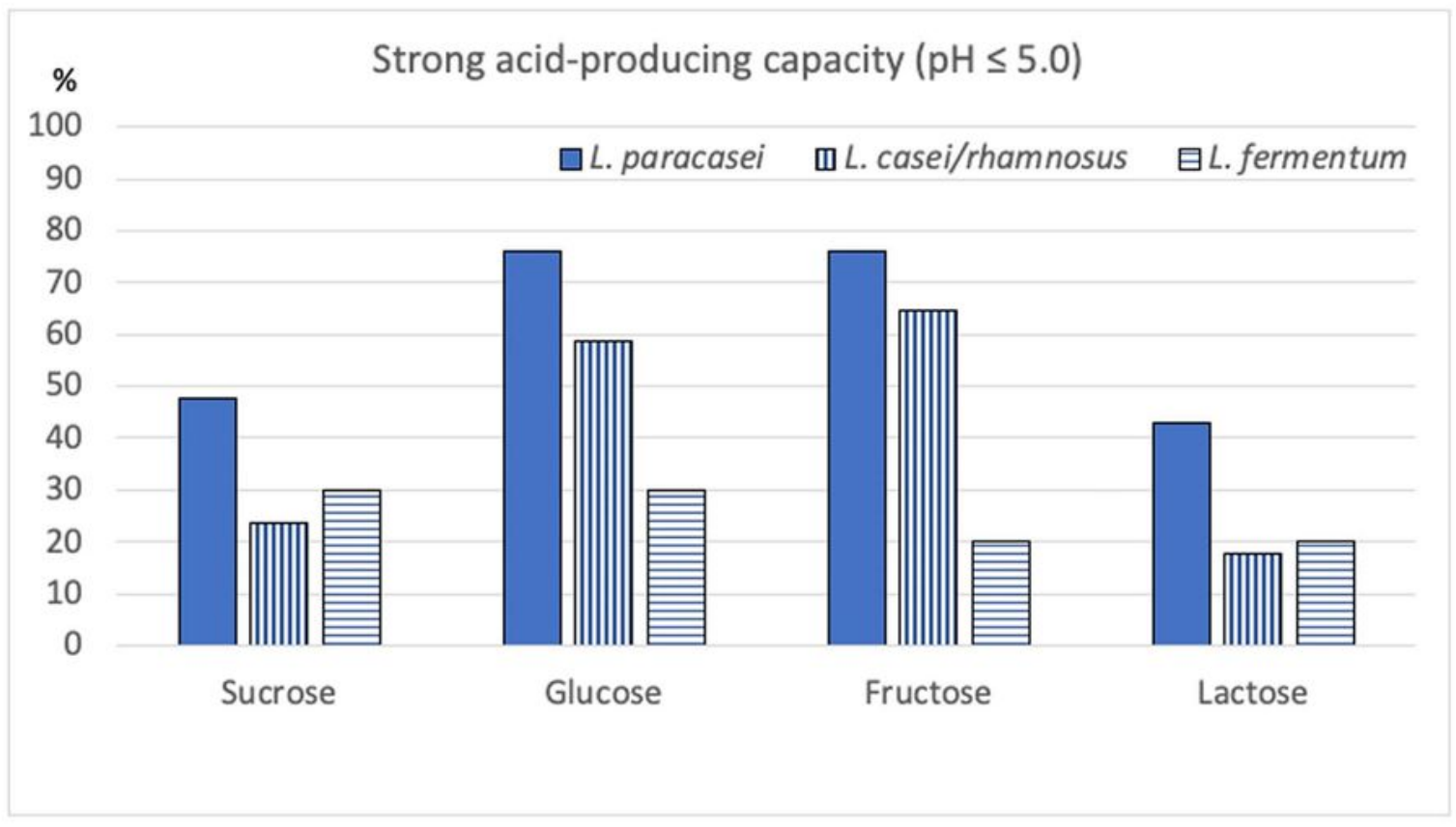

Figure 1

1a. Strong acid-producing capacity for all L. paracasei $(n=21)$, L. casei/rhamnosus $(n=17)$ and $L$. fermentum $(n=10)$ strains irrespective of time-point for collection. 1b. Strong/weak acid-producing capacity for all L. paracasei $(n=21)$, L. casei/rhamnosus $(n=17)$ and L. fermentum $(n=10)$ strains irrespective of time-point for collection. 


\section{$2 a$}

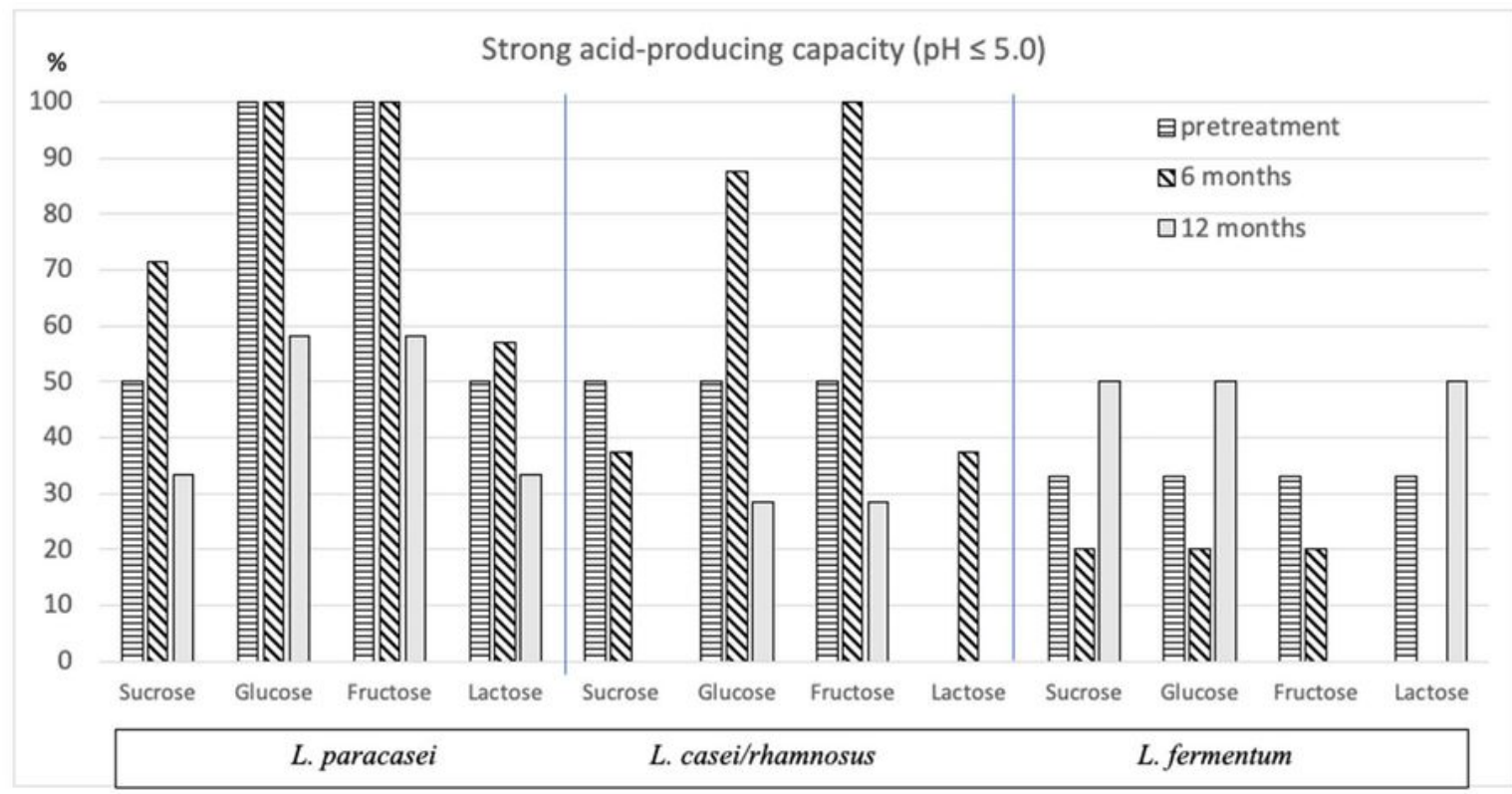

\section{$2 b$}

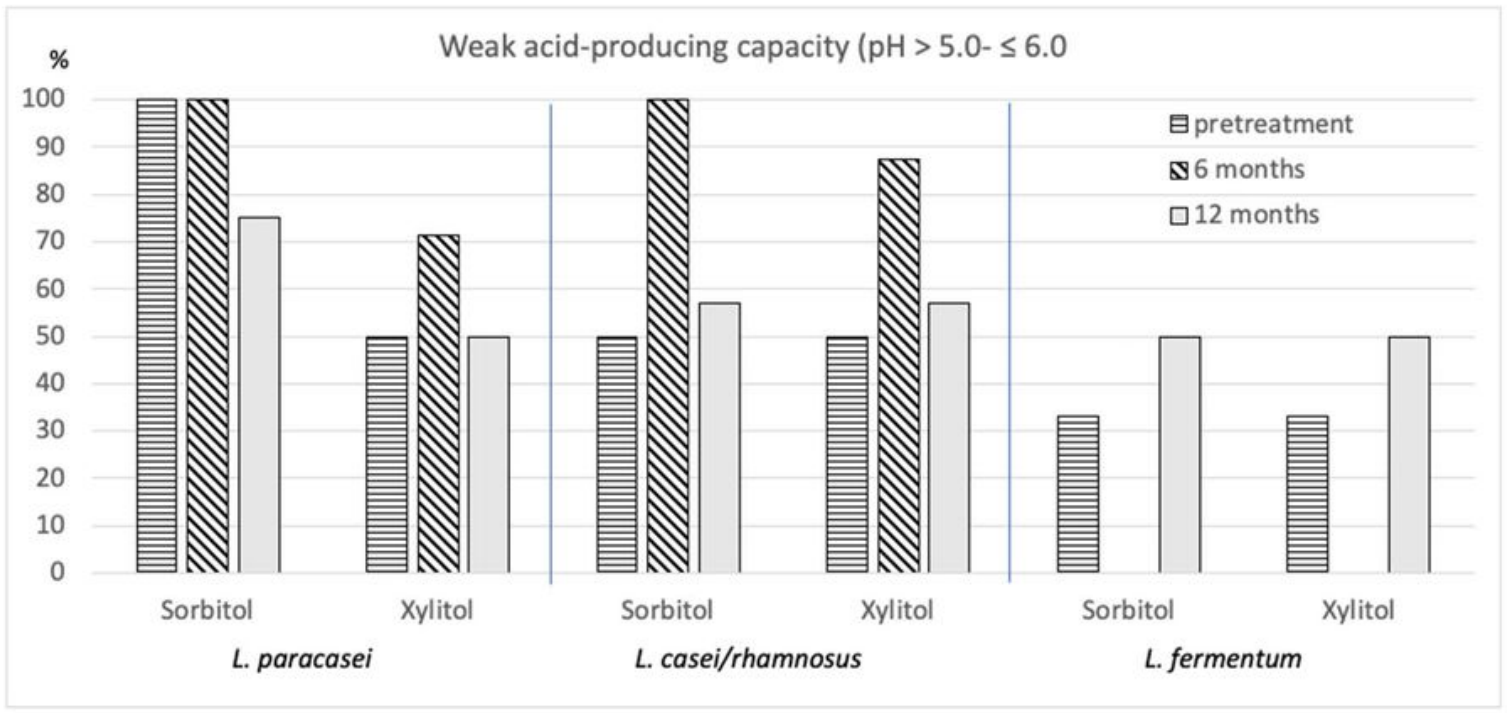

Figure 2

2a. Strong acid-producing capacity using sugars for strains of $L$. paracasei (pretreatment $n=2,6$ months $n=7,12$. months $n=12$ ), L. casei/rhamnosus (pretreatment $n=2,6$ months $n=8,12$ months $n=7$ ) and $L$. fermentum (pre-treatment $n=3,6$ months $n=5,12$ months $n=2$ ). 2b. Weak acid-producing capacity for strains of L. paracasei (pretreatment $n=2,6$ months $n=7,12$ months $n=12$ ), L. casei/rhamnosus (pretreatment $n=2,6$ months $n=8,12$ months $n=7$ ) and L. fermentum (pre-treatment $n=3,6$ months $n=5$, 
12 months $n=2$ ) collected pre-treatment, 6 months and 12 months post treatment. L. paracasei includes one strain showing strong acid-producing capacity at 6 months post treatment and 3 strains at 12 months post treatment.

\section{Supplementary Files}

This is a list of supplementary files associated with this preprint. Click to download.

- supplement1.png

- supplement2.png

- supplement3.png

- supplement4.png 